\title{
3D Elevation Model of Lunar Muti-Craters on Single Charge-coupled Device Image
}

\author{
Junlin Wang ${ }^{12}$ \\ Faculty of Information Technology \\ Macau University of Science and Technology, Macau ,999078, China \\ E-mail: zifuqiwu@gmail.com \\ Yao Wang \\ Faculty of Information Technology \\ Macau University of Science and Technology, Macau,999078,China, \\ E-mail:513334180@qq. com
}

\section{Bingbin Liu}

Faculty of Information Technology

Macau University of Science and Technology, Macau ,999078,China

E-mail:ketes19890163.com

\section{Xiaolin Tian ${ }^{3}$}

Lunar and Planetary Science Laboratory/Space Science Institute Macau University of Science and Technology, Macau,999078,China

E-mail:xltian@must.edu.mo

During the spaceflight technique development, the information of the lunar geography plays an important role in the space exploration. The algorithm in this paper gives a new way to simulate the lunar muti-craters from single $\mathrm{CCD}$ (Charge-coupled Device) image. With the parameter of the lunar environment, the simulation model can work well on processing the lunar highlands CCD images. Comparing with the Tsai's Shape from shading algorithm, the simulation results of the new algorithm have shown more details of craters topography.

CENet2015

12-13 September 2015

Shanghai, China

\footnotetext{
${ }^{1}$ Speaker

${ }^{2}$ Correspongding Author

${ }^{3}$ This work is supported by the Science and Technology Development Fund of Macao (No. 059/2013/A2)
} 


\section{Introduction}

Impact crater is an important and basic geomorphic feature on the lunar surface. Also there are several types of impact crater landforms on the lunar surface, such as the Large-scale Ejecta Ridges, Lunar Highlands and the Highlands-Mare Boundaries. In order to make the landing of probes successfully, the geomorphic information of different impact craters is necessary. Therefore Building the 3-D lunar map plays an important role in the mission planning and navigation of the probe. [1-3]

Usually due to low sampling frequency of laser range finder on the orbiter, the Digital Elevation Map (DEM) is often with low resolution for the lunar remote image. By the Shapefrom-shading (SFS) algorithm, elevation information can be obtained from the CCD images with high resolutions. SFS can be usually used in the 3D reconstruction and can only base on a single input image. Proposed by B.K.P and Horn first[4], the brightness values of the image pixels are used as input of the chromaticity model function designed upfront in this algorithm, and solution of equation is elevation information.

On the other hand, the SFS algorithm usually needs some constraint conditions. Several samples have been simulated by the traditional algorithm, but the results had lost some geomorphic information of the impact craters. The algorithm can be improved by considering the environment parameters on the lunar surface. We have built our model and use the parameters from the lunar surface environment. Large and small craters can be simulated at same time. The results give a well vision of the lunar muti-craters geography.

\section{New algorithm}

In this section, the characteristics and parameters under are described. A series of operations have been used to simulate the craters of the lunar muti-craters from the single CCD image. The flowchart of the new algorithm proposed is shown in Fig. 1 below.

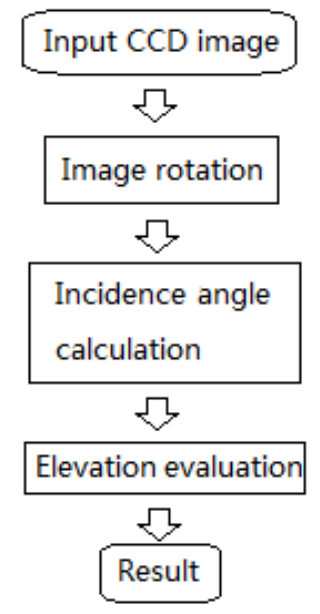

Figure 1: The flowchart of the new algorithm proposed

\subsection{Illumination Analysis}

The traditional SFS algorithm needs some constraint conditions. The Oren-Nayar reflectance model [5] which is a famous model of the SFS, gives a specific example of the illumination equation. But the model in this paper has considered the parameters of the environment on the lunar surface [6]. The craters on the lunar surface always have a dark part when the altitude angle of the sun (angle of incidence is the angle from normal incidence, which is 0 when the sun is the highest) is smaller than the gradient. Then the simplify equation of the illumination can be determined as the flowing 


$$
L=M \frac{\cos \left(\frac{\pi}{2}-\alpha-2 \beta\right)}{\cos \beta}
$$

where $\beta$ is the altitude angle of the sun and $\alpha$ is the gradient. There are also other variables should be known before restore the crater geomorphology. And in the equation 1, the $\mathrm{M}$ is the median gray scale value of the input image. This equation gives the relationship between the gradient with the gray scale $\mathrm{L}$ which is in the input CCD image.

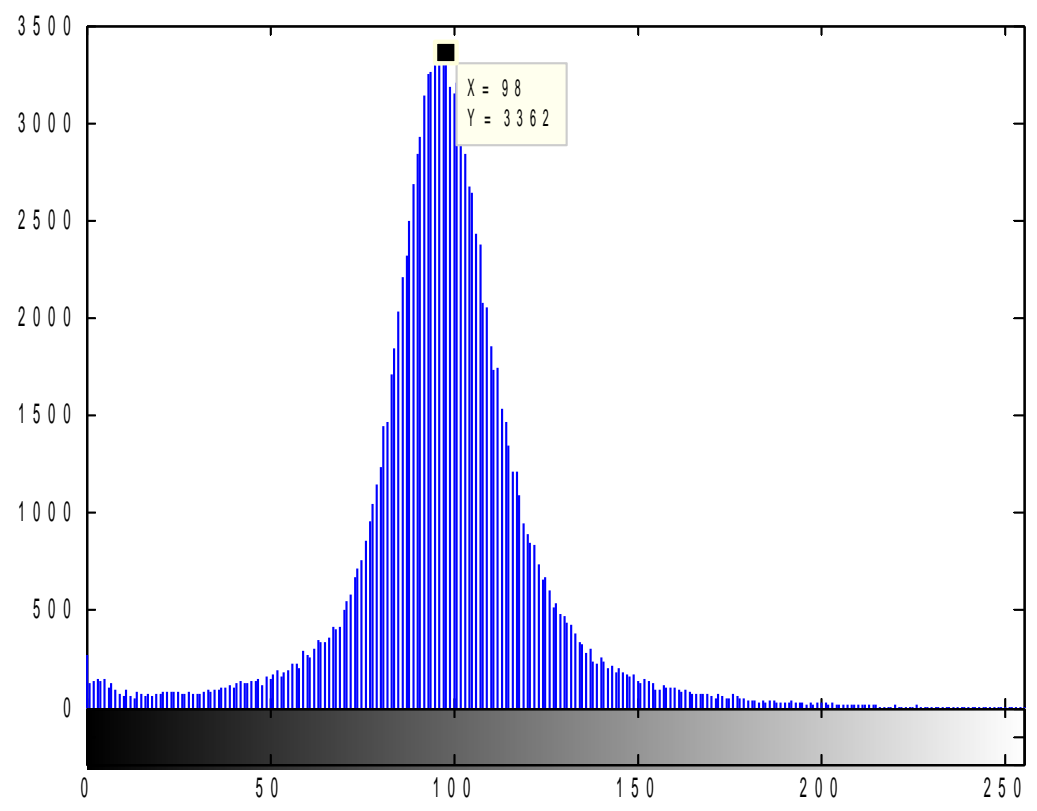

Figure 2: The median gray scale value

Fig.2 shows the gray scale histogram of a CCD image. From the figure, we can find the median gray scale of the CCD image. This parameter gives the flat ground information of the CCD image, and it makes the model can to work on different CCD images. Also form the Lunar source book [7], the gradients of the craters on the lunar high lands are usually less than $45^{\circ}$. So the CCD image can be simulated by the algorithm in this paper since the gradients of the gray scales can be found in a single corresponding equation.

\subsection{Elevation Evaluation}

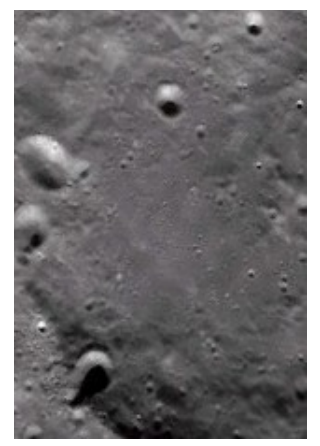

Figure 3: Input figure 


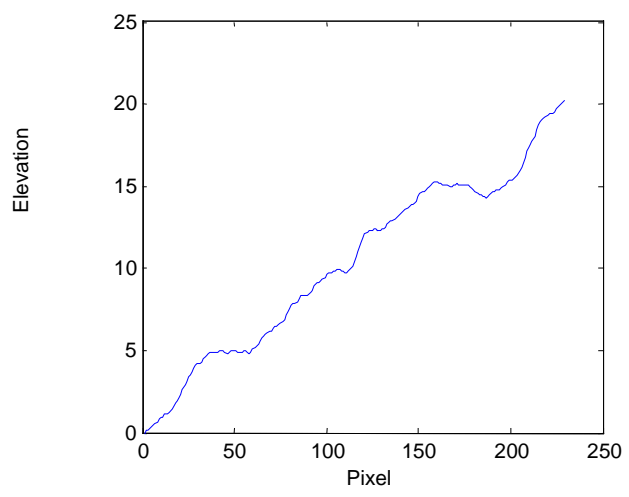

Figure 4 : Edge elevation

The elevation rebuilding is different from the single crater rebuilding. The input image has a large size lunar landform. And the elevation of the beginning column of the image will give a great effect to the simulation result. So the column's elevation should been found before the elevation rebuilding. By using the equation 1, we can get the elevation value for the beginning rebuilding column.

\section{Experimental Results and Discussion}

The purpose of the algorithm is to simulate the muti-craters geomorphology. Two training samples and six testing samples have been selected to test this new algorithm. The simulation results are also compared with the classic SFS algorithm created by Tsai[8], and the result by the algorithm in this paper is better than the Tsai's.

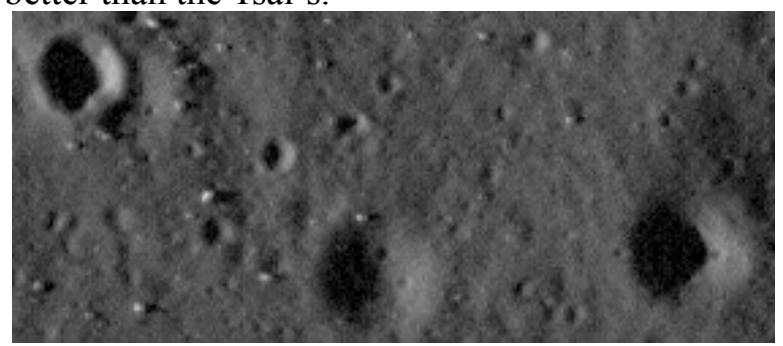

Figure 5: Origin CCD image 1

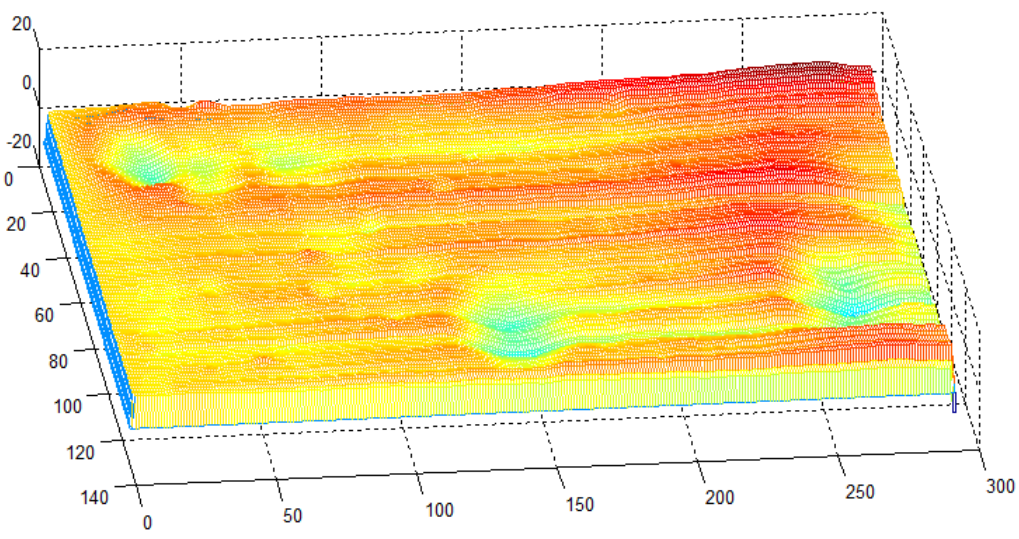

Figure 6: Result image by the algorithm in this paper 


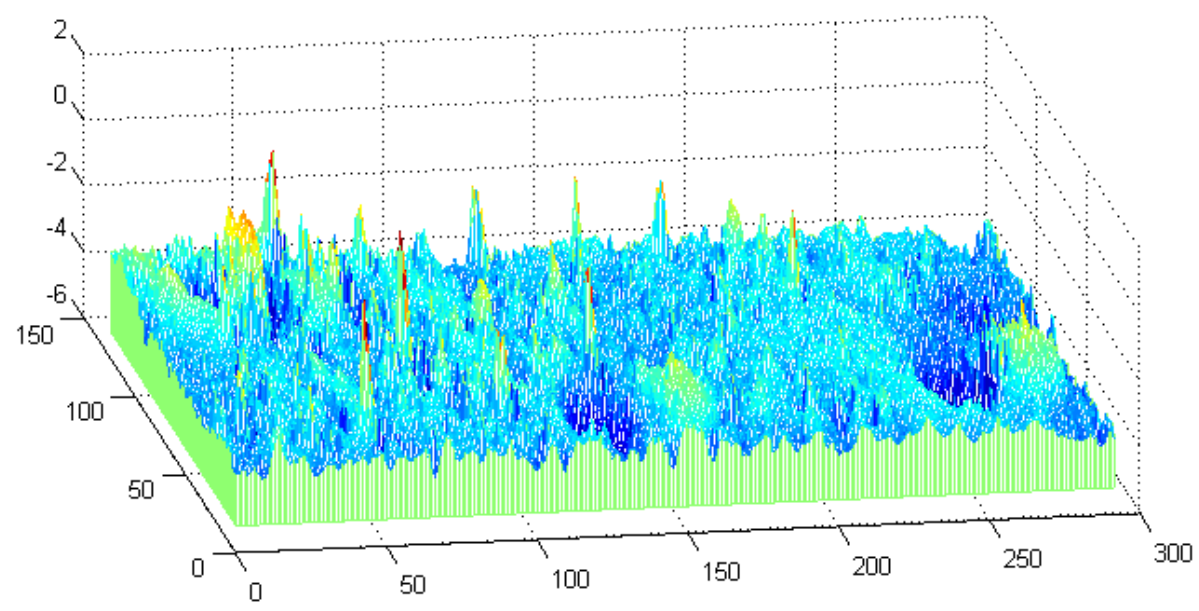

Figure 7 : Result image by the Tsai algorithm

In this experiment, the direction of the light source has been given as $30^{\circ}$. The input image should be changed as the light source angle is $30^{\circ}$. So we specify the dark part of the craters must be on the left of the whole image, by spinning the single CCD images, the image can be adjusted in place. The transverse tectonic structure begins from the left of the single CCD images. According to the testing results, the simulation model of the algorithm in this paper can almost rebuild the geomorphology of the craters. And the Tsai algorithm has not done well in the dark part and light part of the craters, it can only simulate half of the whole crater. The result has been shown in Fig. 8. The Tsai algorithm can't rebuild the part which has a large grayscale because it hasn't considered the source's angel and the dark part and light part's gradient.
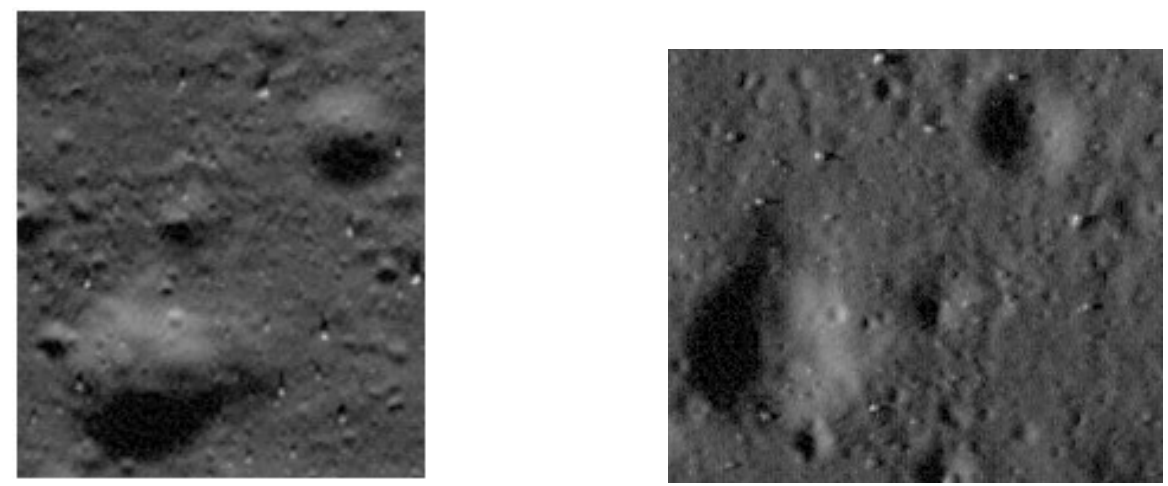

Figure 8 : Origin CCD image 2(Before spinning and after spinning)
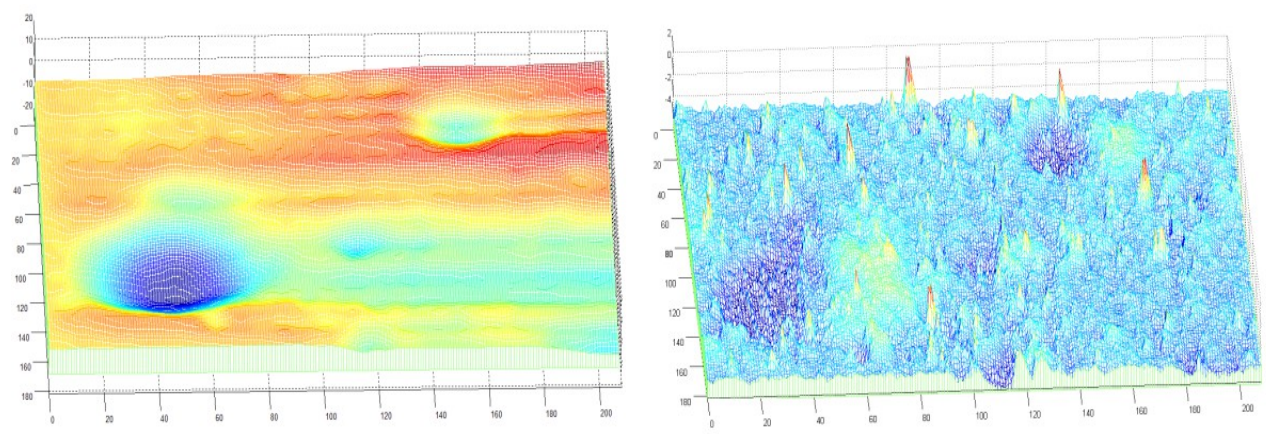

Figure 9: Processed by the algorithm in this paper

Figure 10: Processed by the algorithm the Tsai algorithm 


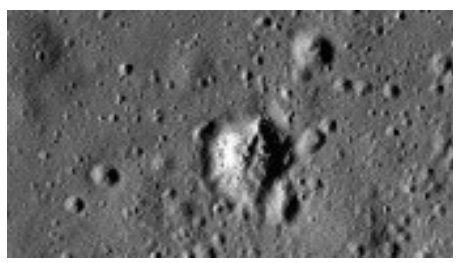

Figure 11: Origin CCD image 3
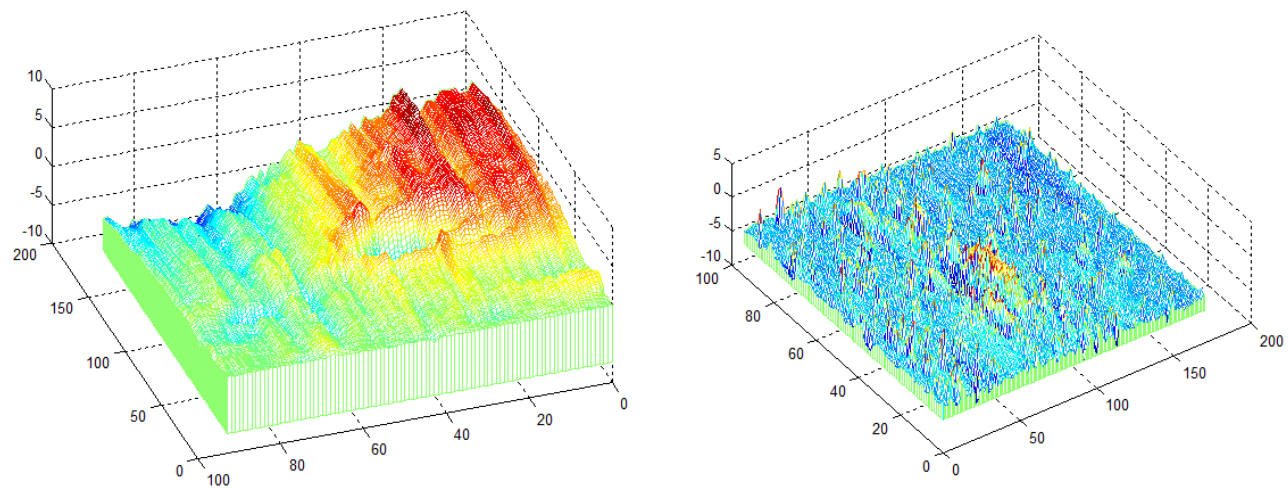

Figure 12: Processed by the algorithm in this paper Figure 13: Processed by the algorithm the Tsai algorithm

This problem leads to a result which has distortion. In the other side, the result of the algorithm in this paper can rebuild the geomorphology clearly and the Tsai algorithm's result can also rebuild the ground which includes muti-craters of the lunar surface, but there is a lot noisy in the simulation result. Comparing with the result in the other paper [9-11], the algorithm in this paper can rebuild the whole crater in the input CCD image and has less noisy in the results.

\section{Conclusion}

This paper has referred to the Oren-Nayar reflectance according to the lunar environment, which makes the model more suitable for the lunar surface. The simulation results are better than the existing algorithm before the new algorithm can show more details of the craters.

But this new algorithm still has some limitations. Such as the algorithm not work very well in a large size input CCD image, the result will be effect by the input CCD image size. We will try to remove these limitations in our future works.

\section{References}

[1] Y. Jia, J. X. Chen, H. G. Zhang. Key Technology Analysis of Lunar Rover, Spacecraft engineering, 2006,16(03):54-58.

[2] Williams D. R, Grayzock E. J. The lunar data projectrestoration of Apollo data for future lunar exploration.Lunar and Planetary Science, 2006, 37(06):1187-1224.

[3] K. Xu, Y. Zhou, J. S. Li, F. Teng, L. V. Liang. A Lunar Landing Area Select Method Based on Single Image. Journal of Geomatics Science and Technology, 2012, 29(2): 127-130.

[4] B. K. P. Horn, The variational approach to shape from shading MJ Brooks. Computer Vision, Graphics, and Image Processing, 1986

[5] L. B Wolff, S. K. Nayar, Oren M. Improved diffuse reflection models for computer vision. International Journal of Computer Vision, 1998, 30(1): 55-71. 
[6] J. Wang, J. Li, B. Liu, et al. 3D Simulation Model of Lunar Crater Based on Single CCD Image. First International Conference on Information Science and Electronic Technology (ISET 2015). Atlantis Press, 2015.

[7] Heiken G, Vaniman D, French B M. Lunar sourcebook: A user's guide to the Moon[M]. CUP Archive, 1991.

[8] P. S. Tsai, M. Shah. A fast linear shape from shading. Computer Vision and Pattern Recognition, 1992. Proceedings CVPR'92, 1992 IEEE Computer Society Conference on. IEEE, 1992: 734-736.

[9] K. Xu, Y. Zhou, .F Teng, J.-S. Li. 3D Simulation of Lunar Surface Based on Single Image. Computer Engineering, 2012, 38(14): 269-271.

[10] X. Xu, A. Xu, S. Liu, Z. Xu. Research on Lunar Crater Topography Reconstruction under Perspective Projection. 2014 (2): 37-41.(In Chinese)

[11] X. Xu, A. Xu, S. Liu, Z. Xu. 3D reconstruction algorithm for lunar crater based on new SFS. Science of Surveying and Mapping. (2014): 017. (In Chinese) 